\title{
Getting To Know the Person Behind the Illness - The Significance of Interacting with Patients Hospitalized in Forensic Psychiatric Settings
}

\begin{abstract}
Aim. To describe what nurses want to accomplish in relationships with patients who are hospitalized in forensic psychiatric settings.

Background. Relationships between staff and patients in forensic psychiatric settings should be grounded in trust and confidence, and the patients need opportunities for emotional reconciliation. However, relationships can be challenging for nurses, who sometimes distance themselves from patients' expressions of suffering. The role of forensic mental health nurses is nebulous, as are the prescriptives and the implementation of nursing practices. Design. Qualitative descriptive design.
\end{abstract}

Methods. In-depth interviews with five nurses who all work in forensic psychiatric settings. Results. We present a descriptive analysis of what nurses want to accomplish in relationships with patients who are hospitalized in forensic psychiatric settings. The results are presented in two main categories: a) getting to know the person behind the illness and b) making a difference. Conclusion. Care in forensic psychiatry needs to shift towards a more long-term view of the role of nursing, focusing less on the traditional and stereotypical identity of the productive nurse and more on the care given when nurses slow down and take the time to see the patients as individuals. Establishing trusting relationships with patients in forensic psychiatric settings is viewed as a less oppressive way to control patients and guide them in directions that are preferable for the nurses as well as for society.

Relevance to clinical practice. Nurses may use simple strategies in their daily practice such as sitting on the sofa with patients in order to establish trust. We stress that nurses should abandon 
This is the pre-print version of the article Salzmann-Erikson, M., Rydlo, C. \& Wiklund Gustin, L. (2016). Getting to know the person and making an impact - The significance of interacting with patients who are hospitalized in forensic psychiatric settings. Journal of Clinical Nursing, 25(9-10), 1426-1434. 10.1111/jocn.13252

policing roles and custodial activities in favour of guiding principles that promote individual recovery, treatment and health-promoting care.

Keywords: forensic psychiatric care, identity, interactions, mental health nursing, nursepatient relationship, psychiatric nursing.

\section{Summary Box}

- Caring in forensic psychiatry needs to shift focus towards a slower, more holistic approach to the process of recovery, away from a traditional and stereotypical identity of the productive nurse.

- Accomplishing trusting relationships with patients in forensic psychiatric settings is viewed as a less oppressive way to guide patients in a direction that is preferable for the nurses as well as for society. 


\section{Introduction}

The Swedish government has written guidelines describing the underlying values of forensic mental health nursing (FMHN) (SOU 2006:100). The document emphasizes the importance of offering patients the same service and quality as they encounter in other parts of the health care system with regards to human worth, respect for self-agency and integrity to the degree that it is possible. An important difference is that in court-mandated psychiatric care the patients do not have the right to choose their caregivers. The guidelines also emphasize that court-mandated psychiatric care should be individualized based on the patient's unique problems and needs. According to a Swedish qualitative interview study, one way to fulfil the individualization and empathetic approach to the relationship is to "see the person behind the crime" (Kumpula \& Ekstrand, 2013). Furthermore, the relationship between staff and patients should be grounded in trust and confidence, creating an environment where the patient has an opportunity for emotional reconciliation. However, research demonstrate that such values are not always implemented. Morrison (1990) used the term culture of toughness to describe staff's macho-like behaviour, in which staff identified their roles to be handling patients by controlling them, adopting a police-like role and putting on a show of authority. If nurses fail to separate the individual and the crime, they risk creating a distance between patients and staff as patients are likely to perceive staff as guards rather than people that they can establish helpful and trusting relationships with (Hörberg, 2008; Maguire, Daffern \& Martin, 2014). Rather than fulfilling the intentions of the guidelines, such cultures tend to be fostering rather than caring, as nurses become emotionally distant from forensic patients. Hence, there is a need for further research that addresses nurse-patient interactions within FMHN.

\section{Background}


The constitution in Swedish law gives guidance for courts to assign individuals who have committed crimes, as a consequence of severe mental disorders, to forensic care within special psychiatric care facilities (SFS 1991:1129). When individuals are assigned to forensic care, this is always grounded in a thorough psychiatric examination that has concluded that the suspect was affected by a severe psychiatric condition during the act of the crime (SFS 1991:1129). Even though these persons are cared for in secure settings due to a decision by a court of law, the focus for forensic care is not to "do time" as a penalty. Rather the intention is grounded in the rehabilitation values in which the individual should be able to be re-integrated into society and gain self-agency.

The benchmark for treatment in forensic psychiatry is grounded on some of the basic principles guiding other care contexts: to improve quality of life, alleviate symptoms and promote recovery. In addition, forensic psychiatry also includes risk aspects in which treatment aims to reduce the risk the individual poses to society (Eastman, Adshead, Fox, Latham \& Whyte, 2012). In general health care, patients must consent to treatment; however, when patients are forced into treatment by a court of law and, in addition, have a skewed perception of the seriousness of the committed act (see Spencer \& Tie, 2013), a patient's consent to treatment becomes complex. Within forensic psychiatry, psychological interventions are considered to be important in order to change the individual's internal processes. The interventions aim to affect the patient's way of thinking, re-enact emotions, and, by extension, develop social skills in order to be re-aligned with society’s values and norms (Dennis et al., 2012).

Nursing approaches and recovery in forensic settings. Research by Askola et al. (2015) argues for the importance of patients' verbalizations of their offenses as means to making sense of their behaviours and thus contributing to patients' recoveries. Interventions that focus on re-enacting emotions can also be demanding for patients and may result in suffering that is 
expressed as violent behaviour and anger (Pereira, Furegato \& Pereira 2005). Another approach to forensic nursing is described by Cook, Phillips and Sadler (2005); rather than focusing on reenacting, they highlight how patients need to reclaim their stories as a part of recovery. This is not possible without close interactions with other people.

A hallmark in psychiatric nursing is to establish alliances and trusting relationships in order to make a change that will help and direct patients' processes toward health and recovery (Borg \& Kristiansen, 2004; Buchanan-Barker \& Barker, 2005; Denhov \& Topor, 2012; Peplau, 1994; Shanley \& Jubb-Shanley, 2007). The processes that take place between nurses and patients have been emphasized by a wide range of nursing theorists. Hildegard Peplau published her interpersonal relational theory, which emphasizes that the nurse-client relationship is at the heart of the nursing process (Peplau, 1992). Moreover, Miller, Leadingham, McKean and McManus (2010) state that the primary role in FMHN is grounded in the tenets of basic nursing. However, this kind of relationship can also be challenging for nurses in forensic care, who sometimes distance themselves from patients' expressions of suffering or consider them as inevitable (Vincze, Fredriksson \& Wiklund Gustin, 2015). Furthermore, in FMHN, the nurse bridges the criminal justice system and the tenets in health care. Hence, the role of FMH nurses is nebulous, as are the prescriptives and the ways FMHN is practiced (Hörberg, 2008).

There are various descriptions of desirable characteristics for FMH nurses. For example, Miller et al. (2010) stressed the importance of interpersonal skills and the ability to think critically. Furthermore, Bowring-Lossock (2006) also highlighted the importance of interpersonal skills and stressed personal maturity and the establishment of relationships with forensic patients. Gildberg, Elverdam and Hounsgaard (2010) studied characteristics of staff interactions within forensic mental health inpatients and the significance the interactions had for the patients. They pointed out that relationships may be paternalistic, as nursing practice includes 
This is the pre-print version of the article Salzmann-Erikson, M., Rydlo, C. \& Wiklund Gustin, L. (2016). Getting to know the person and making an impact - The significance of interacting with patients who are hospitalized in forensic psychiatric settings. Journal of Clinical Nursing, 25(9-10), 1426-1434. 10.1111/jocn.13252

rule enforcements, confrontations, and setting limits; in this sense the relationship had little therapeutic relevance for patients. However, they also stressed that care is quality-dependent due to relational and personal characteristics, as staff express positive attitudes towards the patients by being honest, relaxed, and laid-back and showing interest in the patients. Another study addressed the positive outcomes of interacting with patients in psychiatric forensic settings in an empathetic manner; the therapeutic relationships were more likely to be preserved by patients in comparison to an authoritarian approach (Maguire et al., 2014). Just as Maguire et al. reported on authoritarian styles, Hörberg (2008) reported similar findings, which she identified as 'noncaring', involving interactions between patients and staff that do not support the patient's care. Instead, such non-empathetic styles add more suffering. Patients from Hörberg's study spoke of a climate among staff described as commanding, humiliating and uninterested, and at times staff were described as taking out their anger on patients. Such a culture has negative effects on patients' experiences of hospitalization and treatment. In addition, if nurses keep a distance from patients, these patients are likely to perceive them as guards rather than people that they can establish supportive and trusting relationships with (Hörberg, Brunt \& Axelsson, 2004).

Even though previous studies to some extent have demonstrated the importance of establishing relationships between nurses and patients in psychiatric nursing, these studies tend to focus on nurses' skills and behaviours rather than on nurses' reflections on these interactions. As presented above, nurse-patient interactions can be especially challenging in forensic settings, and in line with Vincze et al. (2015) it can be concluded that forensic nurses sometimes fail to “do good", even when they have good intentions. Therefore it is important to further explore what FMH nurses describe as significant when establishing relationships with patients who are hospitalized in forensic psychiatric settings. Hence, this study looks at FMH nurses' descriptions 
This is the pre-print version of the article Salzmann-Erikson, M., Rydlo, C. \& Wiklund Gustin, L. (2016). Getting to know the person and making an impact - The significance of interacting with patients who are hospitalized in forensic psychiatric settings. Journal of Clinical Nursing, 25(9-10), 1426-1434. 10.1111/jocn.13252

of what they want to accomplish from relationships with patients who are hospitalized in forensic psychiatric settings.

\section{Methods and Materials}

In this specific paper, we focused our qualitative analysis on the in-depth narratives of five nurses who all work in forensic psychiatric settings. We used qualitative descriptions as described by Sandelowski (2000). This method was considered to be well-suited for our study, as it aims to "answer to questions of special relevance to practitioners and policy makers" (Sandelowski, 2000, p. 337). Qualitative description was also chosen because of the method's strength in providing straightforward descriptions of the participants' responses without using a philosophical framework. Basically, qualitative description includes three main steps: becoming familiarized with the data, reducing the data to thematic units in relation to the research question and, finally, re-representing the data in a descriptive summary of the content of data.

\section{Setting and Participants}

Out of six Regional Forensic Psychiatric Hospitals in Sweden, one was chosen due to its geographical distance from the financial resources of the project. The hospital holds over one hundred patient beds and is separate from the county's general hospital. With the help of the clinical head supervisor at the university associated with the hospital, potential participants were provided with a written information sheet, thereby formally asking them to participate in the study. Registered nurses working in a forensic psychiatric setting were selected; no specific exclusion criteria were applied. The clinical head supervisor purposely asked nurses that represented maximum variation in age, gender, and work experience in different wards within the hospital (Patton, 2002). In a first round, six nurses were asked and all agreed to participate. When scheduling time for the interviews, one of the nurses was not able to participate due to a leave of absence. In the second round, the clinical head supervisor asked additional nurses, but 
This is the pre-print version of the article Salzmann-Erikson, M., Rydlo, C. \& Wiklund Gustin, L. (2016). Getting to know the person and making an impact - The significance of interacting with patients who are hospitalized in forensic psychiatric settings. Journal of Clinical Nursing, 25(9-10), 1426-1434. 10.1111/jocn.13252

no one was interested. The participants were comprised of three men and two women, ages 25-

50 years old, who all had worked at the hospital for several years.

\section{Data Gathering}

The interviews took place at the participants' workplace during working hours in an undisturbed room. At the interview session, they received oral information about the study and were asked to sign an informed consent paper. All interviews were audio recorded. The length of the interviews ranged between 54-69 minutes. A trigger question was asked: "I am not at all familiar with the hospital. Can you please tell me about the ward that you are working in?" This question tended to ease the atmosphere in the interview room. Several kinds of question were asked. For example, the interviewers used descriptive questions such as, "How do you socialize with the patients?" and "What is nursing?" From that point, we asked open-ended questions and follow-up questions based on the participants' narratives (Gorden, 1992). The interviews covered the domains of caring and nursing in forensic psychiatry. For example, we asked questions about their views of being professional nurses and how they interacted with patients. In formulating the interview questions in situ, we were guided by Spradley (1979) and Gorden (1992). The interviews ended with the question, "Is there anything else that you want to tell me or is there something that I should have asked but I have not?"

\section{Data Analysis}

First, the interviews were transcribed verbatim. According to the first step in qualitative description as described by Sandelowski (2000), we became familiarized with the data by repeated readings of the transcriptions. In a sense this could be termed as naive reading, as coding was not practiced at this stage. This initial step helped us to get an overall understanding of the data set. In the next step, the texts were more thoroughly read, and they were given codes based on the content. Along with this coding procedure, notes were written on a separate 
This is the pre-print version of the article Salzmann-Erikson, M., Rydlo, C. \& Wiklund Gustin, L. (2016). Getting to know the person and making an impact - The significance of interacting with patients who are hospitalized in forensic psychiatric settings. Journal of Clinical Nursing, 25(9-10), 1426-1434. 10.1111/jocn.13252

spreadsheet in order to follow up on themes, hunches and clues that were identified in the data, such as where, when and why interactions between nurses and patients occurred. From the codes and memos, themes were constructed that could provide an answer in accordance with the aim of the study. The themes and their relevancies to the data set were then discussed within the research group. Due to the nature of qualitative description, we did not search for underlying and latent meanings and kept the level of interpretation at a minimum. Rather, our goal was to summarize the main themes of the informants' descriptions and re-represent them in a way that fit the data (Sandelowski 1998; 2000). In our presentation of the results, excerpts from the data were chosen that would provide a good representation of each theme.

\section{Ethical Considerations}

The project was reviewed and approved by the Regional Ethical Review Board (dnr 2011/214). The informants received both written and verbal information about the study and their rights to decline participation and to withdraw at any time. The integrity of the informants was protected as far as possible in that no personal characteristics were presented.

\section{Results}

We present a descriptive analysis of what nurses want to accomplish from relationships with patients who are hospitalized in forensic psychiatric settings. The results are presented in two main categories: a) getting to know the person behind the illness and b) making a difference.

\section{Getting to Know the Person Behind the Illness}

Based on their experiences of caring for patients in forensic psychiatry, the participants explained the importance of getting to know the patients as people. From their experiences, they spoke of different patients they had established relationships with over the years as a result of daily interactions. First and foremost, participants stressed that establishing a relationship with patients was pivotal. In doing so, it is possible to separate the person from the crime committed 
This is the pre-print version of the article Salzmann-Erikson, M., Rydlo, C. \& Wiklund Gustin, L. (2016). Getting to know the person and making an impact - The significance of interacting with patients who are hospitalized in forensic psychiatric settings. Journal of Clinical Nursing, 25(9-10), 1426-1434. 10.1111/jocn.13252

in order to see the person "behind the diagnosis" and "behind the crime". A driving principle was to overcome the focus on daily ward-related and practical tasks and prioritize the time spent with patients. One participant narrated this belief:

"The first thing I usually attempt is to establish a relationship,

contact. Because without a relationship, there is no care, I believe.

You can't just be present in a unit and go about your duties and tasks

because that isn't care... (I need to) establish a relationship and see

what kind of person it is that has come in to me..."

Another participant, who also stressed the significance of getting to know the patients as people, drew an example from when he once had been a supervisor to a nursing student. He concluded that nursing student was often given practical tasks to conduct as part of the curriculum in the university. However, he questioned the value of this focus on doing; rather he emphasized the importance of learning the value of being with the patients in order to establish relationships with patients.

"I: No, I got her to stop herself, to stand up... take it easy. She threw herself into her school assignment here on day 2 so that she could just be here the rest of the week and I... no.. there, see the sofa in there? Where five patients are sitting? 'Yes' You should sit there. (laugh) 'Just sit?' No you can talk, of course... (laugh). In other words she wanted to do (the assignments)... and I forced her to do the other part... (laugh). ... the students see more and more of the person behind the disease. And if they didn't sit on the sofa during those hours, they'd never do it so they'd just see a room number, a name and a diagnosis and possibly a status update (laugh) and that's a sad picture of a person. The fun, complex picture... 
This is the pre-print version of the article Salzmann-Erikson, M., Rydlo, C. \& Wiklund Gustin, L. (2016). Getting to know the person and making an impact - The significance of interacting with patients who are hospitalized in forensic psychiatric settings. Journal of Clinical Nursing, 25(9-10), 1426-1434. 10.1111/jocn.13252

or the interesting picture of a person, that's the one you get when you sit down and hang out and you see... when you see the person for real."

The excerpt from the interview promotes the sofa as a tool to make it possible to get to know the patients as people, which was presented as the most profound nursing task. The sofa became a materialized artefact, making leisurely dialogues with patients possible in order to establish contact. Furthermore, the sofa facilitated social occasions, which provided opportunities to override the objectification of patients and to avoid reducing them to a room number, a name and a diagnosis.

Another participant narrated the importance of actively taking responsibility for getting to know the person through interacting, as this facilitates the establishment of a relationship. She believed that there was an "old truth" in psychiatric care recommending that patients should be left alone during the initial phase of hospitalization. She spoke with irony about these kinds of old beliefs, such as the often-heard sayings that nurses should "give patients time to settle in" and "wait out the patient". In her view, as she matured into a more senior nurse, she had adopted a critical position toward such beliefs, as she could not defend such passivity in care. Rather she claimed that the moral responsibility of the nurse is to actively take initiative and advocated for the necessity of establishing relationships with patients in order to get to know them.

"I: Then it's possible that the patient gets to settle into the care unit and then it's... that you're supposed to wait out the patient (Mmmm) and from experience I've seen that you have patients that will never make contact (Mmmhmm), should that patient just be kept lying in his room? (Mmmm) Is that okay? No, not by my estimation today (Uh uh). I think that seven years ago I wouldn't have dared to take up this discussion or gotten into an argument about it (Uh uh) ... to contradict more experienced co-workers and colleagues." 
Her beliefs about care stressed the significance of taking active responsibility for the patients' interests. The value of overcoming passivity in care can be understood as significant, as the beliefs were so strong that she considered confronting her colleagues when they held opposing views, particularly in Swedish culture, which looks down on confrontation.

In order to get to know the patients as people, participants told of several strategies for leisurely social activities that encouraged this. These strategies included taking daily outdoor walks, playing Yahtzee, or cleaning the patient's room. What the activities have in common are the interaction and the notion of doing something together with the patients, both of which facilitate the relationship and bridge the distance in the predetermined roles of "patient" and "staff". One participant differentiated between "shallow" and "profound" conversations. She stressed the importance of having leisurely chats with patients in front of the TV about everyday themes but also emphasized the need for more in-depth conversations in order to "reach the essence" of the patients' problems. In order to get to know the patients, nursing observations were expressed as crucial; however, as yet another participant expressed, the observations must also be followed by nursing action.

"But the most important thing is still to be able to draw in someone... that has needs, that is, we talk about inviting someone in to a conversation, and they don't always say yes, but still you show that 'listen, you looked a little down during the meal, how are you feeling right now? Do you want to talk or go for a walk or something? Or can I sit with you for a bit?",

From the excerpt in the interview, the participant viewed that her nursing observations of a patient that looked down became an opening to actively engage with the patient.

\section{Making a Difference}


This is the pre-print version of the article Salzmann-Erikson, M., Rydlo, C. \& Wiklund Gustin, L. (2016). Getting to know the person and making an impact - The significance of interacting with patients who are hospitalized in forensic psychiatric settings. Journal of Clinical Nursing, 25(9-10), 1426-1434. 10.1111/jocn.13252

The participants also narrated the significance of interacting with patients when they were trying to have an impact on the patients with their interactions. In contrast to the first category which put focus on being with a person, the category of making a difference implied making progress on the patient's health. Helping a patient make progress was interpreted as complex nursing, as it is a prerequisite to an established relationship between the nurse and the patient. Making a difference was the result of engaging with the patients, which extended over long periods of time. The participant emphasized that "making a difference" was important, as it incorporated caring values in their nursing; otherwise the hospitalization would merely be imprisonment/confinement. In addition to the focus on caring when establishing relationships with the patients, the aspect of doing was also identified in the data. In one excerpt in an interview, the participant stated that patients were likely to express challenging behaviours, and a goal in care was to impact the patient's behaviours.

"It's important that when you have these tough patients that might take lots of energy or that can be threatening and that can hit hard [...] that you go through difficult situations, for example, you had to strap down someone so then you go through it afterwards, what you did well in the group or if you could have done something different and you even go through that with the patient and the person's experience of being strapped down, they themselves can recognize what led up to all that... why they ended up strapped down and how we staff have handled it, if there was something we did. [...] you don't just strap someone else and say 'see you' and then get them to go away or something. You go through this and it maybe can lead to next time maybe the person doesn't end up strapped in. [...] and then you go through it and then the patient can maybe say, 'yes, maybe it was because of that. It was probably 
This is the pre-print version of the article Salzmann-Erikson, M., Rydlo, C. \& Wiklund Gustin, L. (2016). Getting to know the person and making an impact - The significance of interacting with patients who are hospitalized in forensic psychiatric settings. Journal of Clinical Nursing, 25(9-10), 1426-1434. 10.1111/jocn.13252

that that set me off, I handled it this way without thinking.' Or it can also be, 'it was that staff member, he said this and that got me to think about this, I don't like his way of interacting with me'. something like that."

In this example, having an impact included dialoguing with the patient about the situation in which the nurses wanted to accomplish a change. Since hospitalization in forensic psychiatry spans over several years, making a difference happened over time. This long-term care perspective included both having an impact in the short run, as in the example above, but also making a difference in the long run. Regarding the long run, participants narrated that caring was about impacting the patients' ways of thinking and making them reflect on their previous ways of living. For example, many patients had had criminal lifestyles, and in such, their lifestyles were considered to be deeply rooted in their identities. Making a difference in the long run was viewed as caring, as it addressed the intersection between behaviours and lifestyles that impacted negatively upon the individual. Moreover, nursing that made a difference involved providing patients with alternatives that are seen as meaningful by the patients, where the alternative would make it worth a change. Another excerpt from an interview demonstrates how caring might impact the patients:

"I can take this patient that we had for nine or ten months. He was always, you know, unfriendly... without perspective... and the whole time we tried with medicine and everything. A lot of the staff were really tired of it and almost hit a wall because this person demanded so much... he took so much energy from everyone and threatened both staff and patients, he was ungrateful. He served his sentence and had a date for release. When he was about to be released, I thanked him that last evening, and he got tears in his eyes and hugged me and said 'thank you, thank you, thank you'. When they were going to leave him off, 
This is the pre-print version of the article Salzmann-Erikson, M., Rydlo, C. \& Wiklund Gustin, L. (2016). Getting to know the person and making an impact - The significance of interacting with patients who are hospitalized in forensic psychiatric settings. Journal of Clinical Nursing, 25(9-10), 1426-1434. 10.1111/jocn.13252

he started to cry and then you could see what the people around him had meant

[...] You feel like we've done something good, we've worked with the person

that maybe no one else could deal with but he was thankful in the end."

In the long run, caring was also associated with abiding patients' expressions of mental illness.

As viewed in the excerpt above, the staff dealt with a patient who was disagreeable and

expressed what was viewed as unpleasant behaviour. However, being there for the patient in the

long run resulted in the patient expressing gratitude.

\section{Discussion}

\section{Methodological Considerations}

This study is a small-scale study comprised of in-depth interviews with five nurses. Even though the quantitative number of participants is low, Kvale (1996) claims that $15+/-10$ is sufficient in qualitative research. Furthermore, as this kind of research is not a question of numbers but of meanings, it is important to reflect on whether or not the data are meaningful rather than focusing on the number of participants. We have interpreted the data set and value content as meaningful for an analysis using qualitative description. The data set includes the narratives of the participants' experiences when working with patients in forensic settings, and several examples were given from their everyday work as nurses. We are aware that this data set does not cover all aspects of caring and nursing in forensic psychiatry, but as participants gave in-depth descriptions of their experiences of interacting with the patients, different aspects of establishing relationships with patients were prominent in the data. We argue for the potential value of the results in this study, as it gives a first-hand glance at what FMH nurses focus on as important when establishing relationships in this specific context. Furthermore, this study provides guidance for further research using more thorough research methods 
This is the pre-print version of the article Salzmann-Erikson, M., Rydlo, C. \& Wiklund Gustin, L. (2016). Getting to know the person and making an impact - The significance of interacting with patients who are hospitalized in forensic psychiatric settings. Journal of Clinical Nursing, 25(9-10), 1426-1434. 10.1111/jocn.13252

such as fieldwork, including participant observations. At the time when the interviews were conducted, the informants expressed a positive attitude towards our study, which reflects the significance of the study aims and the interview questions.

\section{The role of FMH Nurses}

The purpose of this study was to look at FMH nurses' descriptions of what they want to accomplish in relationships with patients who are hospitalized in forensic psychiatric settings. The findings reveal that nurse-patient interactions could be understood as means to achieve their goals in nursing. First, interaction was a strategy to get to know the patient as a person and, when such a relationship was established, nursing also included having an impact on the patients' behaviours, thinking and lifestyles. The findings are in accordance with the guidelines of Government Official Reports (SOU 2006:100), and from this perspective participants' reflections express mature, self-aware and reflexive insights in their practices of FMHN. This is also in line with Eastman et al.'s (2012) emphasis that forensic psychiatry should be based on the same principles as other care contexts, including valuing the patient as a person in his/her own right. This is indeed evident in the first category, getting to know the person. The second category, making a difference, appears as implicitly related to Eastman and colleagues' claim that forensic psychiatry also aims to reduce the risk the individual poses to society. The fact that our results did not address the angle of risk explicitly does not necessarily indicate that those aspects were not considered to be important for the participant from a wider perspective. Rather, making a difference was related to progress in patients' thoughts and behaviours in such a way that patients could adapt to the current situation and to society. Thus, the narratives focus on what counts as significant in a "here and now" perspective. 
Few studies have accounted for inquiries that emphasize the significance of interactions between nurses and patients within FMHN. Vincze et al. (2015) conducted a study to explore nurse-patient interactions within FMHN in order to understand and approach patients' suffering. Vincze and colleagues found that some FMH nurses asserted the relevance of nurses' emotional presence in order to ease the patients' suffering as well as their genuine interest in the patients' life worlds. Another facet in their study was the nurses' abilities to let the patient set the pace when it came to conversations about the crime. Their study supports many of the findings in our study. For example, our analysis suggests that to establish relationships with patients, the aspects of being with the patients were accentuated. For example, sitting on the sofa was used as a strategy to initiate and have leisurely dialogues to get to know a patient as a person and therefore be able to see the individual behind the crime.

However, even though we argue for the value of being with patients, we also acknowledge that it can be a challenge for nurses. Thus nurses might distance themselves from patients in order to cope with such challenges (Jacob, Holmes \& Buus, 2011; Vincze et al. 2015). Furthermore, Salzmann-Erikson, Lützén, Ivarsson and Eriksson (2008) stress that it takes courage, will and stamina to care for patients with demanding expressions as a result of mental illness. Thus the role and identity of FMH nurses highly influence the quality in care. In general, due to limited resources in the health-care system, the work of nurses tends to be more task oriented, at the cost of possibilities to develop intimate relationships with patients (Chambers \& Ryder, 2009). This sheds light on the risk that "getting to know the person" and "making a difference" in the patients' thoughts, behaviours and lifestyles could be used as means to protect oneself and control patients in order to adjust them rather than to promote recovery from the person's perspective (Barker, 2003; Breeding, 2014). Hence, from this perspective nurses' descriptions of what they want to accomplish from relationships with patients in forensic 
psychiatric settings also could be viewed as less oppressive ways to control patients and guide them in directions that are preferable for the nurses as well as society. This is in line with the claim that the humanistic values that underpin nursing theories are in conflict with the highly specialized field of forensic nursing (Jacob et al., 2008). This gap between theoretical ideals and the grim reality of practices, such as guarded and humiliating attitudes among staff (Hörberg, 2008), can also confuse FMH nurses' roles and identities.

Much of the identity of a nurse is defined by the activities s/he engages in, such as taking blood samples, giving injections and performing wound care. Hörberg (2008) described the role of the FMH nurse as nebulous, although Miller et al. (2010) presented a more clear view in her theoretical framework of FMHN, in which interpersonal skills and critical thinking were accentuated. Willetts and Clarke (2013) argue that little research has been conducted on the social identity of nurses and its correlation to their professional identity. Such ambiguity may contribute to devaluing the nurse's role, or, in the words of Newman, Smith, Pharris and Jones (2008), "Without a clear sense of our nursing identity and the meaning of our mission to society, we have no value or purpose other than to support and promote the practice of medicine" (p. E25). Stereotyping exists among mental health nurses (De Carlo, 2007) as well but is less cemented, and what a nurse should engage in is less well-defined concerning forensic psychiatry. We hypothesize that such unclear roles and identity are an explanation as to why other studies report contradicting view of the everyday in forensic care settings. In contrast to our findings, previous research has demonstrated a more dismal view when reporting on interactions between patients and nurses in psychiatric settings and forensic psychiatry. For example, Morrison (1990) reported on a "culture of toughness" to describe staff's macho-like behaviour and Hörberg (2008) found similar descriptions with only "pockets of good care” (p. 208). Furthermore, Mason and Chandley (1990) also viewed nurses' practices in forensic psychiatry to be likened to 
This is the pre-print version of the article Salzmann-Erikson, M., Rydlo, C. \& Wiklund Gustin, L. (2016). Getting to know the person and making an impact - The significance of interacting with patients who are hospitalized in forensic psychiatric settings. Journal of Clinical Nursing, 25(9-10), 1426-1434. 10.1111/jocn.13252

societal policing. In yet another, more recently published doctoral dissertation, Hörberg (2008) reported that FMHN is to a large extent disciplining, where punishment and reward are the tools being used by staff in order to create a structure in which the patients are expected to adapt to.

In contrast there are research revealing that forensic caring cultures can change (Cook et al., 2005). Our results present the value of overcoming the role of a task-oriented nurse to become a being-oriented nurse in order to be present. This is in line with Boeck's (2014) claim that presence as a core value could enhance the integration of relationship-centred modalities in nursing care. We argue that whether nurses take time to closely engage with patients or distract themselves is a result of both the culture of the setting and the identity of the nurse within that culture. Based on our study, we argue that caring in forensic psychiatry needs to shift focus towards a slower, more long-sighted culture and away from a traditional and stereotypical identity of the productive nurse. Being aware that our findings could be understood as means to oppress patients in a "soft and gentle" way, we still claim that times are changing. Even though we do not have any reason to doubt the trustworthiness in the interviews, we cannot be sure that the participants act and behave in everyday care in the way they have narrated their work. But, importantly, they describe a caring ideology that is in accordance with ethical values that promote the individual interest of the patient's well-being. As a result, a participant talked about her own maturity as a nurse, and she felt confident in arguing against older, more entrenched beliefs. These findings were similar to what Gillespie and Flowers (2009) discussed: that the older, custodial culture is being replaced by new ethical values that account for individual recovery, treatment and health-promoting care.

\section{Conclusions}

Caring in forensic psychiatry needs to shift focus towards a slower, more long-sighted culture, away from a traditional and stereotypical identity of the productive nurse. 
This is the pre-print version of the article Salzmann-Erikson, M., Rydlo, C. \& Wiklund Gustin, L. (2016). Getting to know the person and making an impact - The significance of interacting with patients who are hospitalized in forensic psychiatric settings. Journal of Clinical Nursing, 25(9-10), 1426-1434. 10.1111/jocn.13252

Accomplishing trusting relationships with patients in forensic psychiatric settings is viewed as a less oppressive way to control patients and guide them in a direction that is preferable for the nurses as well as for society.

\section{Relevance to Clinical Practice}

We assert that FMH nurses should reflect on the need to move beyond their roles as taskdriven nurses and become being-oriented. The results indicate that FMHN may make use of simple strategies such as sitting on a sofa with patients in order to move toward a caring ideology that is in accordance with ethical values and promotes the individual interest in the patient's well-being. As such, FMH nurses are able to get to know the patients, and through the establishment of trusting relationships, they will gain the ability to have an impact on doing progress for the patient's health, both in the short and long run.

\section{Conflict of Interests}

The authors declare that they have no conflicts of interests. 


\section{References}

Askola, R. A., Louheranta, O., Paavilainen, E., Åstedt-Kurki, P., Soininen, P., Putkonen, H., \& Nikkonen, M. (2015). Forensic psychiatric patients' narratives of their offense. Issues in Mental Health Nursing, 36(3), 162-170.

http://dx.doi.org/10.3109/01612840.2014.969391

Barker, P. (2003). The tidal model: Psychiatric colonization, recovery and the paradigm shift in mental health care. International Journal of Mental Health Nursing, 12(2), 96-102. http://dx.doi.org/10.1046/j.1440-0979.2003.00275.x

Boeck, P. R. (2014). Presence. A concept analysis. SAGE Open, 1-6. http://dx.doi.org/10.1177/2158244014527990

Borg, M., \& Kristainsen, K. (2004). Recovery-oriented professionals: Helping relationships in mental health services. Journal of Mental Health, 13(5), 493-505.

Bowring-Lossock, E. (2006). The forensic mental health nurse - a literature review.

Journal of Psychiatric and Mental Health Nursing, 13(6), 780-785.

http://dx.doi.org/10.1111/j.1365-2850.2006.00993.x

Breeding, J. (2014). Practicing Szasz: A psychologist reports on Thomas Szasz's influence on his work. SAFE Open, 1-12. http://dx.doi.org/10.1177/2158244014551715

Buchanan-Barker, P., \& Barker, P. (2005). Observation: The original sin of mental health nursing? Journal of Psychiatric and Mental Health Nursing,12(5), 541-549. http://dx.doi.org/111/j.1365-2850.2005.00876.x

Chambers, C., \& Ryder, E. (2009). Compassion and caring nursing. Oxford: New York: Radcliffe Publishing.

Cook, N. R., Phillips, B. N., \& Sadler, D. (2005). The tidal model as experienced by patients and 
This is the pre-print version of the article Salzmann-Erikson, M., Rydlo, C. \& Wiklund Gustin, L. (2016). Getting to know the person and making an impact - The significance of interacting with patients who are hospitalized in forensic psychiatric settings. Journal of Clinical Nursing, 25(9-10), 1426-1434. 10.1111/jocn.13252

nurses in a regional forensic unit. Journal of Psychiatric and Mental Health Nursing, 12(5), 536-540. http://dx.doi.org/10.1111/j.1365-2850.2005.00872.x

De Carlo, K. (2007). Ogres and Angels in the madhouse: Mental health nursing identities in film. International Journal of Mental Health Nursing, 16(5), 338-348. http://dx.doi.org/10.1111/j.1447-0349.2007.00488.x

Denhov, A., \& Topor, A. (2012). The components of helping relationships with professionals in psychiatry: Users perspective. International Journal of Social Psychiatry, 58(4), 417424. http://dx.doi.org/10.1177/0020764011406811

Dennis, J. A., Khan, O., Ferriter, M., Huband, N., Powney, M. J., \& Duggan, C. (2012). Psychological interventions for adults who have sexually offended or are at risk of offending. Cochrane Database of Systematic Reviews, 12, CD007507.

Eastman, N., Adshead, G., Fox, S., Latham, R., \& Whyte S. (2012). Oxford specialist handbooks in psychiatry: Forensic psychiatry. Oxford, New York, NY: Oxford University Press.

Gildberg, F. A., Elverdam, B., \& Hounsgaars, L. (2010). Forensic psychiatric nursing: A literature review and thematic analysis of staff-patient interaction. Journal of Psychiatric and Mental Health Nursing, 17(4), 359-368. http://dx.doi.org/10.1111/j.1365-2850.2009.01533.x

Gillespie, M., \& Flowers, P. (2009), From the old to the new: Is forensic mental health nursing in transition? Journal of Forensic Nursing, 5(4), 212-219. http://dx.doi.org/10.1111/j.1939-3938.2009.01056.x

Gorden, R. (1992). Basic interviewing skills. Peacock Publications, IL.

Hörberg, U. (2008). Att vårdas eller fostras. Det rättspsykiatriska vårdandet och traditionens grepp. (English: To be the subject of care or the object of 
This is the pre-print version of the article Salzmann-Erikson, M., Rydlo, C. \& Wiklund Gustin, L. (2016). Getting to know the person and making an impact - The significance of interacting with patients who are hospitalized in forensic psychiatric settings. Journal of Clinical Nursing, 25(9-10), 1426-1434. 10.1111/jocn.13252

correction. Forensic psychiatric care and the power of tradition) (Unpublished doctoral dissertation). Acta Wexionensia No 133/2008: Växjö.

Hörberg, U., Brunt, D., \& Axelsson, Å. (2004). Client's perspective of client-nurse relationships in local authority psychiatric services: A qualitative study. International Journal of Mental Health Nursing, 13(1), 9-17. http://dx.doi.org/10.1111/j.14470349.2004.00303.x

Jacob, J. D., Holmes, D., \& Buus, N. (2008). Humanism in forensic psychiatry: The use of the tidal nursing model. Nursing Inquiry, 15(3), 224-230. http://dx.doi.org/10.1111/j.14401800.2008.00420.x

Kumpula, E., \& Ekstrand, P. (2013). 'Doing things together': Male caregivers' experiences of giving care to patients in forensic psychiatric care. Journal of Psychiatric and Mental Health Nursing, 20(1), 64-70. http://dx.doi.org/10.1111/j.1365-2850.2012.01887.x

Kvale, S. (1996). Interviews-An introduction to qualitative research interviewing. Thousand Oaks, CA: Sage.

Maguire, T., Daffern, M., \& Martin, T. (2014). Exploring nurses' and patients' perspectives of limit setting in a forensic mental health setting. International Journal of Mental Health Nursing, 23(2), 153-160. http://dx.doi.org/10.1111/inm.12034.

Mason, T., \& Chandley, M. (1990). Nursing models in a special hospital: A critical analysis of efficacy. Journal of Advanced Nursing, 15(6), 667-673. http://dx.doi.org/10.1111/j.13652648.1990.tb01889.x

Miller, C. L., Leadingham, C., McKean, J. R., \& McManus, C. (2010). Forensic nursing: An emerging competency for contemporary practice. Teaching and Learning in Nursing, 5(3), 98-103. http://dx.doi.org/10.1016/j.teln.2010.01.001 
This is the pre-print version of the article Salzmann-Erikson, M., Rydlo, C. \& Wiklund Gustin, L. (2016). Getting to know the person and making an impact - The significance of interacting with patients who are hospitalized in forensic psychiatric settings. Journal of Clinical Nursing, 25(9-10), 1426-1434. 10.1111/jocn.13252

Morrison, E. F. (1990). The tradition of toughness: A study of nonprofessional nursing care in psychiatric nursing. Journal of Nursing Scholarship, 24(2), 32-38. http://dx.doi.org/10.1111/inm.12113

Newman, M. A., Smith, M. C., Pharris, M. D, \& Jones, D. (2008). The focus of the discipline of nursing revisited. Advances in Nursing Science, 31(1), E16-E27.

Pereira, M. A. O., Furegato, A. R. F,. \& Pereira, A. (2005). The lived experience of long-term psychiatric hospitalization of four women in Brazil. Perspectives in Psychiatric Care, 3, 124-132. http://dx.doi.org/10.1111/j.1744-6163.2005.00010.x

Peplau H. E. (1994) Psychiatric mental health nursing: Challenge and change. Journal of Psychiatric and Mental Health Nursing, 1(1), 3-7. http://dx.doi.org/10.1111/j.1365-2850.1994.tb00002.x

Peplau, H. E. (1992). Interpersonal relations: A theoretical framework for application in nursing practice. Nursing Science Quarterly, 5 (1), 13-18. http://dx.doi.org/10.1177/089431849200500106

Salzmann-Erikson, M., Lützén, K., Ivarsson, A-B., Eriksson, H. (2008). The core characteristics and nursing care activities in psychiatric intensive care units in Sweden. International Journal of Mental Health Nursing, 17(2), 98-107. http://dx.doi.org/10.1111/j.1447-0349.2008.00517.x

Sandelowski, M. (1998). Writing a good read: Strategies for re-presenting qualitative data. Research in Nursing \& Health, 21(4), 375-382. http://dx.doi.org/10.1002/(SICI)1098-240X(199808)21:4<375::AIDNUR9>3.0.CO;2-C

Sandelowski, M. (2000). Whatever happened to qualitative description?. Research in Nursing \& Health, 23(4), 334-340. http://dx.doi.org/10.1002/1098- 
Shanley, M. (2007). The recovery alliance theory of mental health nursing. Journal of Psychiatric and Mental Health Nursing, 14(8), 734-743. http://dx.doi.org/10.1111/j.1365-2850.2007.01179.x.

SFS 1991:1129. Lag om rättspsykiatrisk vård [The law on forensic psychiatric care]. Stockholm: Swedish Statute Book.

SOU 2006:100. Ambition och ansvar Nationell strategi för utveckling av samhällets insatser till personer med psykiska sjukdomar och funktionshinder. Statens Offenliga Utredningar. Slutbetänkande av Nationell psykiatrisamordning. Stockholm. Http://www.socialpsykiatrisktforum.nu/media/samlat-nationellpsykiatrisamordning/ambition-och-ansvar-slutbetankande.pdf

Spencer J., \& Tie, A. (2013). Psychiatric symptoms associated with the mental health defence for serious violent offenses in Queensland. Australasian Psychiatry, 21(2), 147152. http://dx.doi.org/10.1177/1039856212470501

Spradley, J. P. (1979). The ethnographic interview. New York, NY: Holt, Rinehart and Winston.

Willetts, G., \& Clarke, D. (2014), Constructing nurses’ professional identity through social identity theory. International Journal of Nursing Practice, 20(2) 164169. http://dx.doi.org/10.1111/ijn.12108

Vincze, M., Fredriksson, L., \& Wiklund Gustin, L. (2015). To do good might hurt bad: exploring nurses' understanding and approach to suffering in forensic psychiatric settings. International Journal of Mental Health Nursing, 24(2),149-157. http://dx.doi.org/10.1111/inm.12116. 